\title{
Study of Synchro-betatron Coupling in IPNS Upgrade RCS
}

\author{
K. C. Harkay \\ Argonne National Laboratory
}

\section{Introduction}

The 2-GeV rapidly-cycling synchrotron (RCS) of the proposed 1-MW spallation neutron source upgrade has 16 dispersion-free straight sections and eight straight sections with a dispersion of approximately $1 \mathrm{~m}$. Six of the ten rf cavities are located in dispersion-free straight sections, and the remainder are located in the non-dispersion-free straight sections. The possibility of exciting synchro-betatron resonances is investigated in this paper. It is found that the effect of the coupling is not important in the RCS.

Synchro-betatron resonances are driven in single beams by position-dependent energy gains from the accelerating fields. A primary cause is the dispersion at the location of the rf cavities. Longitudinal and transverse motions are coupled due to a transverse deflection associated with a particle crossing the if cavity gap. This changes the equilibrium orbit and excites betatron oscillations. The resonance condition for coupling is given by

$$
k v_{x} \pm l v_{y} \pm m v_{s}=n
$$

where $k, l, m$, and $n$ are positive integers, and $v_{x}, v_{y}$ and $v_{s}$ are the betatron and synchrotron tunes, respectively. In proton machines in general, and in the RCS in particular, $v_{s} \ll<v_{x, y}$. Therefore, the synchro-betatron resonances $(m \geq 1)$ manifest as satellites of the integer $(k+l=1)$ resonance, and the resonance condition for coupling becomes

$$
v_{x, y} \pm m v_{s}=n
$$

Here, the \pm signs refer to the sum and difference resonances, respectively.

\section{Growth Rate}

On resonance, according to Suzuki, 1 the maximum growth rate of the energy spread per revolution, $\delta(\Delta E / E)$, and the maximum betatron oscillation amplitude change per revolution, $\delta x$, are given by 


\section{DISCLAIMER}

This report was prepared as an account of work sponsored by an agency of the United States Government. Neither the United States Government nor any agency thereof, nor any of their employees, make any warranty, express or implied, or assumes any legal liability or responsibility for the accuracy, completeness, or usefulness of any information, apparatus, product, or process disclosed, or represents that its use would not infringe privately owned rights. Reference herein to any specific commercial product, process, or service by trade name, trademark, manufacturer, or otherwise does not necessarily constitute or imply its endorsement, recommendation, or favoring by the United States Government or any agency thereof. The views and opinions of authors expressed herein do not necessarily state or reflect those of the United States Government or any agency thereof. 


\section{DISCLAIMER}

Portions of this document may be illegible in electronic image products. Images are produced from the best available original document. 


$$
\begin{array}{cc}
\delta\left(\frac{\Delta E}{E}\right)_{\max }=\frac{\beta^{3}\left|\alpha_{m}\right|}{c p R \beta_{x}} \frac{\phi_{\max }^{m} x}{2^{m}(m-1) !|\eta|(\Delta E / E)} A_{\eta}, & {\left[\mathrm{rev}^{-1}\right]} \\
\delta x_{\max }=\frac{\left|\alpha_{m}\right|}{c p \beta} \frac{\phi_{\max }^{m}}{2^{m} m !} A_{\eta}, & {\left[\mathrm{m} \mathrm{rev}^{-1}\right]}
\end{array}
$$

where $R$ is the average synchrotron radius, $\beta$ and $\gamma$ are the standard time-varying relativistic factors, $\eta=\gamma_{t}^{-2}-\gamma^{-2}$ is the slip factor, $\gamma_{t}$ is the transition energy, $p$ is the beam momentum, $E$ is the beam energy, $x=\sqrt{\varepsilon_{x} \beta_{x} / \pi}$ is the betatron oscillation amplitude, $\beta_{x}$ is the lattice parameter at the cavity, and $\phi_{\max }$ is the maximum phase of the synchrotron oscillations. The Taylor expansion coefficient, $\alpha_{m}$, is given by

$$
\alpha_{m}=\left\{\begin{array}{cc}
-(1)^{m / 2} \sin \phi_{s} & \text { m even } \\
-(-1)^{(m+1) / 2} \cos \phi_{s} & \text { m odd }
\end{array}\right. \text {. }
$$

The parameter $A_{\eta}$ depends on the rf voltage and the dispersion function, and is given by

$$
\begin{gathered}
A_{\eta}=\sqrt{\left(D_{c n}-F_{s n}\right)^{2}+\left(D_{s n}+F_{c n}\right)^{2}} \\
D_{c n}=\sum_{j} e V_{j} \eta_{x j} \cos n \phi_{x j} \quad D_{s n}=\sum_{j} e V_{j} \eta_{x j} \sin n \phi_{x j} \\
F_{c n}=\sum_{j} e V_{j} F_{j} \cos n \phi_{x j} \quad F_{s n}=\sum_{j} e V_{j} F_{j} \sin n \phi_{x j} \\
F=\eta_{x}^{\prime} \beta_{x}+\eta_{x} \alpha_{x}
\end{gathered}
$$

where, for each cavity $j, V$ is the accelerating voltage, $\alpha_{x}, \beta_{x}$, and $\eta_{x}$ are the Courant-Snyder lattice parameters, and $\phi_{x}$ is the betatron phase advance. The sum is over one turn.

\section{Results}

The RCS operates entirely below transition energy ( $400 \mathrm{MeV}-2 \mathrm{GeV}, \gamma_{t}=5.40$ ); therefore, the sum resonance is unstable and the difference resonance is stable. However, growth in the betatron oscillation amplitude may be important even for difference resonances. The largest $v_{s}$ is 0.003 , which occurs at $4 \mathrm{msec}$ in the $25-\mathrm{msec}$ acceleration cycle. Here the synchrotron frequency is $4 \mathrm{kHz}$ and the revolution frequency is $1.2 \mathrm{MHz}$. Using Eqn. (1) and $v_{x}=6.821$ and $v_{y}=5.731$, the minimum orders $m$ for the four-sided RCS lattice 2 are about 60 for the sum (xplane) and about 246 for the difference ( $y$-plane) resonance. The estimated growth rates using 
Eqns. (2) and (3) are vanishingly small for such large orders of the synchro-betatron resonance due to the factorial term in the denominator.

As an illustration, $\delta x$ is evaluated at $4 \mathrm{msec}$. The relevant beam parameters for the RCS are given in Table 1, while the average lattice parameters in the if straight sections are given in Table 2. The RCS lattice for one super-period (out of four) is shown in Figure 1, where the long, if straight sections are marked: L1, L2, ... ,L6. The quadrupoles, QF and QD, the chromaticity-correcting sextupoles, SF and SD, and the harmonic-correcting sextupoles, S1, are also marked.

Table 1. Beam parameters for the RCS at $4 \mathrm{msec}$ in the cycle

\begin{tabular}{cccccccc}
\hline$\beta$ & $\begin{array}{c}p \\
(\mathrm{MeV} / \mathrm{c})\end{array}$ & $\begin{array}{c}x \\
(\mathrm{~mm})\end{array}$ & $v_{s}$ & $|\eta|$ & $\begin{array}{c}\Delta E \\
(\mathrm{MeV})\end{array}$ & $\begin{array}{c}\phi_{\max } \\
(\mathrm{rad})\end{array}$ & $\begin{array}{c}\phi_{s} \\
(\mathrm{deg})\end{array}$ \\
\hline 0.755 & 1080 & 53 & 0.003 & 0.395 & 5 & 1.8 & 26 \\
\hline
\end{tabular}

Table 2. Average lattice parameters in $\mathrm{rf}$ long straight sections

\begin{tabular}{ccrrrrc}
\hline $\begin{array}{c}\text { long } \\
\text { straight } \\
\text { section \# }\end{array}$ & $\begin{array}{c}\left\langle\beta_{x}\right\rangle \\
(\mathrm{m})\end{array}$ & $\left\langle\alpha_{x}\right\rangle$ & $\begin{array}{c}\left\langle\eta_{x}\right\rangle \\
(\mathrm{m})\end{array}$ & $\left\langle\eta_{x}^{\prime}\right\rangle$ & $\begin{array}{c}\phi_{x} \\
(\mathrm{rad})\end{array}$ & $F$ \\
\hline L1 & 6.20 & 1.31 & 0.920 & -0.335 & 2.64 & -0.872 \\
L2 & 6.22 & 1.32 & -0.011 & -0.005 & 4.16 & -0.0456 \\
L3 & 6.21 & -1.32 & -0.041 & -0.013 & 5.01 & -0.0266 \\
L4 & 6.21 & 1.32 & -0.041 & 0.013 & 5.70 & 0.0266 \\
L5 & 6.22 & -1.32 & -0.011 & 0.005 & 6.55 & 0.0456 \\
L6 & 6.20 & -1.31 & 0.920 & 0.335 & 8.08 & 0.872 \\
\hline
\end{tabular}

One rf cavity is placed in each long straight section as follows: (I) L1, L2, L3, L4, L5, L6; (II) L5, L6; and (III) L5, L6. The roman numeral refers to the super-period number. Each of cavity provides $18 \mathrm{kV}$ voltage. A phase advance of $\Delta \phi_{x}=\left(v_{x} / 4\right)(N-1)$ is added to the betatron phase, $\phi_{x}$, listed in Table 2, where $v_{x}=6.821$ is the tune and $N$ is the super-period number. 
For the sum resonance, $n=7$, and the value of $A_{\eta}=50.35 \mathrm{keV}-\mathrm{m}$. The results for the growth in the betatron amplitude are given in Table 3 for synchro-betatron resonance orders up to $m=5$. The mode numbers, $m$, we are concerned about are 60 and 246, and, clearly, the amplitude growth for these numbers is negligibly small.

Table 3. Growth of betatron amplitude for first few orders of $m$

\begin{tabular}{ccc}
\hline$m$ & $\begin{array}{c}\delta x \\
(\mathrm{~mm} / \mathrm{rev})\end{array}$ & $\begin{array}{c}\delta x \\
(\mathrm{~mm} / \mathrm{msec})\end{array}$ \\
\hline 1 & $4.6 \mathrm{e}-3$ & 5.5 \\
2 & $8.3 \mathrm{e}-4$ & 1.0 \\
3 & $4.2 \mathrm{e}-4$ & 0.5 \\
4 & $3.8 \mathrm{e}-5$ & 0.04 \\
5 & $1.2 \mathrm{e}-5$ & 0.01 \\
\hline
\end{tabular}

\section{Conclusion}

In the RCS, the minimum synchro-betatron sum and difference resonance orders are very high. The synchrotron tune would have to increase by two orders of magnitude to cause an appreciable betatron oscillation amplitude due to synchro-betatron coupling. Therefore, the effect of the synchro-betatron resonances can be neglected.

The author would like to thank Y. Cho for helpful discussions and Y.-C. Chae for providing Figure 1.

\section{References}

1. T. Suzuki, "Synchrobetatron resonances driven by dispersion in RF cavities," in Particle Accelerators, 18 (1985).

2. Y. Cho, Lattice for RCS II, Version 2 - Four-Sided Lattice, Argonne National Laboratory Report No. NSA-94-8 (June 1994). 


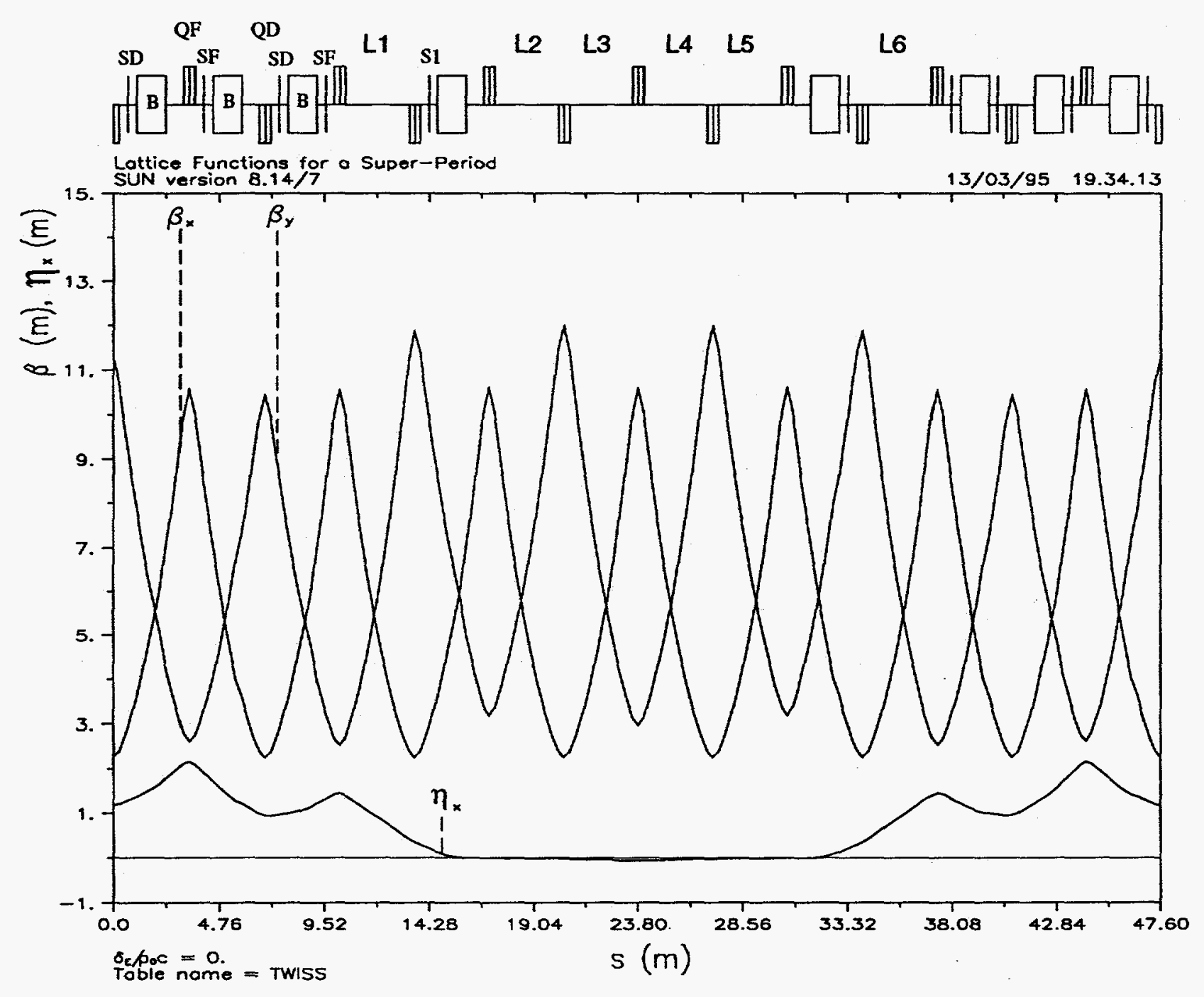

Figure 1. Lattice functions for one super-period in the RCS. 